\title{
Anomaly targets detection of hyperspectral imagery based on wavelet transform and sparse representation
}

\author{
Cheng Baozhia \\ College of Mechanical and Electrical Engineering, Daqing Normal University, Daqing 163712, China
}

\begin{abstract}
The research of anomaly target detection algorithm in hyperspectral imagery is a hot issue, which has important research value. In order to overcome low efficiency of current anomaly target detection in hyperspectral image, an anomaly detection algorithm for hyperspectral images based on wavelet transform and sparse representation was proposed. Firstly, two-dimensional discrete wavelet transform is used to denoise the hyperspectral image, and the new hyperspectral image data are obtained. Then, the results of anomaly target detection are obtained by using sparse representation theory. The real AVIRIS hyperspectral imagery data sets are used in the experiments. The results show that the detection accuracy and false alarm rate of the propoesd algorithm are better than RX and KRX algorithm.
\end{abstract}

\section{Introduction}

In the 21 century, there has been rapid development in the field of remote sensing with the continuous improvement of human ability to understand the material world. Satellite is constantly being launched for military and civilian services with high resolution imagers to ground imaging. In recent years, China has launched high resolution 4 satellite, high resolution 5 satellite and other satellites. Hyperspectral image processing includes theoretical analysis, algorithm research and practical application. The algorithm research mainly focuses on hyperspectral image correction, hyperspectral image fusion, hyperspectral image classification, hyperspectral image pixel unmixed and hyperspectral image target detection. Among them, the anomaly target detection has become a hot spot in recent years. The main reason is that anomaly target detection doesn't need prior information, which can detect the unknown targets in the condition of blind signal.

The key problems are how to distinguish the anomaly targets from the background in hyperspectral image anomaly detection, and how to suppress the background images and estimate them effectively, and how to improve the detection accuracy of the anomaly targets. In 1990, Reed[1] proposed a constant false-alarm rate (CFAR) algorithm based on generalized likelihood ratiotest, which is called RX anomaly target algorithm. The RX is a linear detection algorithm, which lays a kind of framework for anomaly target detection algorithm in hyperspectral images. The local RX anomaly detection was developed in paper[2]. However, neither the global $\mathrm{RX}$ anomaly detection algorithm nor the local RX anomaly detection algorithm consistently describes the real background distribution, which leads to the accuracy reduction of anomaly detection algorithm. Later researchers put forward some improved RX anomaly detection algorithms. For example, the subspace RX[3] anomaly detection algorithm proposed by A.P. Schaum, which improves the original RX algorithm and obtains better anomaly detection results. Kwon[4] proposed nonlinear KRX algorithm[5] based on the nonlinear kernel theory and RX anomaly detection algorithm, which improves the precision of anomaly detection to solving the problem of background nonlinearity. Sparse representation becomes the best data representation after fourier transform and wavelet transform, which is very good to describing distribution of the data. With the introduction of sparse representation theory into hyperspectral image anomaly detection, which is used to process the hyperspectral image data to improve the accuracy of anomaly detection and reduce the false alarm rate. Yuan[6] proposed an anomaly detection algorithm based on local sparse divergence index, which sets spectral divergence index and spatial divergence index, and then it get a decision formula through weighted two divergence indexes. The detection results are obtained by sparse decomposition of hyperspectral images, which can optimize the anomaly detection results of hyperspectral images, but the detection accuracy is not high. Zhang[7] proposed an anomaly detection algorithm for hyperspectral images based on kernel collaborative representation, which obtained good detection results. In recent years, the tensor decomposition method[8] has been introduced into hyperspectral image processing, which has been applied on unmixed and target detection of hyperspectral image, and it doesn't need to transform hyperspectral image data because high-order tensor decomposition can directly

\footnotetext{
${ }^{\mathrm{a}}$ Corresponding author: chengbaozhigy@163.com
} 
process three-dimensional hyperspectral image, and the accuracy of anomaly target detection is high.

In paper, several existing algorithms for hyperspectral image are compared and analyzed. A new anomaly detection algorithm for hyperspectral image is proposed for Wavelet Transform and Sparsity Representation (WTSR), which is simulated and distinguished by the real hyperspectral images.

\section{Method}

\subsection{Wavelet transform}

Wavelet transform can effectively extract information from the signal because it is a local transform in time and frequency domain, which can fully highlight the some aspects characteristics of the solved problem by multiscale analysis for the function or signal through scaling and translating. In recent years, the wavelet transform has been widely applied in hyperspectral images, such as hyperspectral image compression, hyperspectral image denoising, hyperspectral image inversion, hyperspectral image classification and so on. In paper, wavelet transform is used to denoise hyperspectral images. The two-dimensional discrete wavelet transform is applied[9], assuming the discrete wavelet function $\kappa_{j, k}(t)$

$$
\begin{aligned}
\kappa_{j, k}(t) & =\frac{1}{\sqrt{\left|l_{0}\right|}} \kappa\left(\frac{t-m l_{0}^{j} x_{0}}{l_{0}^{j}}\right) \\
& =\frac{1}{\sqrt{\left|l_{0}\right|}} \kappa\left(l_{0}^{-j} t-m x\right)
\end{aligned}
$$

among them, $\quad l, x \in R ; \quad l \neq 0, \kappa$ is admissible.

$l=l_{0}^{j}, x=x_{0}^{j}$ is discretization formula, $j \in Z$.

The discrete wavelet transform coefficients can be expressed as

$$
Q_{j, k}=\int_{-\infty}^{+\infty} f(t) \kappa^{*}{ }_{j, k}(t) d t=<f, \kappa_{j, k}(t)>
$$

its reconstruction formula is

$$
f(t)=Q \sum_{-\infty}^{+\infty} \sum_{-\infty}^{+\infty} Q_{j, k} \kappa_{j, k}(t)
$$

\subsection{Sparse representation for hyperspectral image}

Sparse representation theory has been applied to hyperspectral image processing in recent years. As an effective data representation, it can solve the problem of data analysis. The theoretical models of sparse representation for hyperspectral image data are as follows:

$$
X=D \alpha
$$

where, $\boldsymbol{X}=\left(\boldsymbol{x}_{1}, \ldots \boldsymbol{x}_{i}, \ldots \boldsymbol{x}_{N}\right) \in R^{N}$ is hyperspectral data vector with $L$ spectral bands, $D=\left(d_{1}, \ldots d_{i}, \ldots d_{K}\right) \in R^{N \times K}$ $(N \ll K)$ is Over complete dictionary, $\alpha=\left(\alpha_{1}, \ldots \alpha_{i}\right.$, $\left.\ldots \alpha_{K}\right) \in R^{K}$ is coefficient, $\alpha$ Is sparse Based on sparse representation theory, that is, only a few of the components are nonzero.

Sparse representation can be a good solution to the problem of hyperspectral images. Anomaly target detection of hyperspectral image can be regarded as a special category of two elements, that is, only the background class and the target class in the image. The attribution of the pixel will be determined to the dictionary atoms, which can be used to represent the pixel spectrum, belonging to the background sub-dictionary or the target sub-dictionary because the pixel spectrum in the image can be represented by a linear combination of dictionary atoms[10]. $X$ is arbitrary pixel to be detected, It can be assumed that it is in a space that is shared by the background subspace and the target subspace, its spectrum can be approximately represented as a background sub dictionary $D_{b}$ and target sub dictionary $D_{t}$, Corresponding to the linear combination of the training samples, as follows:

$$
\begin{aligned}
& X \approx \alpha_{1}^{b} d_{1}^{b}+\alpha_{2}^{b} d_{2}^{b}+\cdots+\alpha_{N_{b}}^{b} d_{N_{b}}^{b}+\alpha_{1}^{t} d_{1}^{t} \\
& +\alpha_{2}^{t} d_{2}^{t}+\cdots+\alpha_{N_{t}}^{t} d_{N_{t}}^{t}= \\
& \underbrace{\left[d_{1}^{b} d_{2}^{b} \cdots d_{N_{b}}^{b}\right.}_{D_{b}}] \underbrace{\left[\alpha_{1}^{b} \alpha_{2}^{b} \cdots \alpha_{N_{b}}^{b}\right]^{T}}_{\alpha_{b}}+ \\
& \underbrace{\left[d_{1}^{t} d_{2}^{t} \cdots d_{N_{b}}^{t}\right.}_{D_{t}}] \underbrace{\left[\alpha_{1}^{t} \alpha_{2}^{t} \cdots \alpha_{N_{b}}^{t}\right]^{T}}_{\alpha_{t}}= \\
& D_{b} \alpha_{b}+D_{t} \alpha_{t}=\underbrace{\left.D_{b} D_{t}\right]}_{D}\left[\begin{array}{l}
\alpha_{b} \\
\alpha_{t}
\end{array}\right]=D \alpha
\end{aligned}
$$

where, the dictionary $D$ is $L \times\left(N_{b}+N_{t}\right)$ matrix, which is constituted by the background sub-dictionary $D_{b}$ and target sub-dictionary $D_{t} ; \alpha$ is corresponding to the unknown sparse coefficient weight vector.

The spectral characteristics different between the background pixels and the target pixels, $\alpha=\alpha_{b}+\alpha_{t}$ is sparse coefficient weight vector, $\alpha_{b}$ is background coefficient weight vector, $\alpha_{t}$ is a zero vector. If $X$ is a target pixel, then $\alpha_{b}$ is a zero vector, but $\alpha_{t}$ is sparse. Therefore, the pixel will can be determined, which is the background or target, according to the sparse representation of the pixel coefficient vector of the non-zero coefficient position[11]. Coefficient vector $\alpha$ of solving sparse representation of hyperspectral pixels $X$, namely Solving optimization problems for (3):

$$
\alpha=\arg \min \|\alpha\|_{1} \text { subject to } D \alpha=X
$$

due to the characteristics of sparse vectors $\alpha$, the optimization problem can be solved by using the norm $l_{1}$ minimization problem.

\section{Simulation experiment and analysis}

As is stated above, the key technology and process of the 
algorithm are analyzed. We apply a real hyperspectral images to do anomaly target detection experiments in order to verify the validity and rationality of the proposed algorithm, the real hyperspectral images is part of the San Diego Naval airport, which contains 126 bands(the number of remaining bands after removing the low-information bands), the wavelength range is $0.4 \sim 1.8$ $\mu \mathrm{m}$, the spatial resolution is $3.5 \mathrm{~m}$, and the spatial size is $100 \times 100$ pixels. this part of the image is selected as the data source of the anomaly detection algorithm is that it contains 38 anomaly targets by research and analysis. The $20^{\text {th }}$ band for hyperspectral image and the corresponding real anomay target distribution are shown in Figure 1. In order to compare the advantages and rationality of the proposed algorithm, which is compared with the classical RX algorithm[1] and KRX algorithm[4].

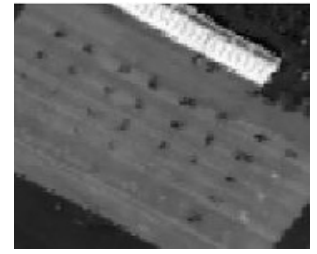

(a)

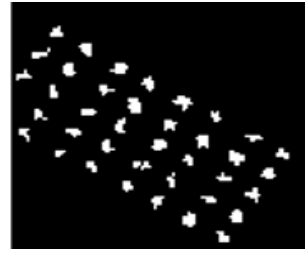

(b)
Figure 1. The hyperspectral image and corresponding targets as simulation, (a) The $20^{\text {th }}$ band hyperspectral image of $100 \times 100$ pixels,(b) The truth anomaly targets distribution

Firstly, wavelet transform is used to denoise the original hyperspectral image data, Figure 2(a) is the result of the first low-pass filtering. Most of the high-frequency noise is filtered out, but there is still a certain amount of high-frequency noise compared with the original hyperspectral image. Figure 2 (b) is the result of the second low-pass filtering. On the basis of the first denoising, the high frequency noise is filtered again, and the denoising effect is better, but the image quality is slightly worse than the original hyperspectral image. In general, the noise interference of the background image is suppressed by compared with the original hyperspectral image. Then, the image is detected by sparse representation.

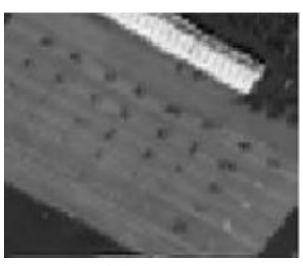

(a)

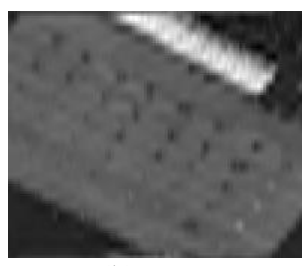

(b)
Figure 2. the $20^{\text {th }}$ band hyperspectral image data after first and second low pass filtering noise reduction, (a) the result of the first low pass filtering, (b) the result of second low pass filtering

The RX and KRX anomaly target detection algorithms are simulated for analysis the effectiveness of the proposed algorithm. The results are shown in Figure 3. In the simulation process, the total number of selected pixels is 400, which can be obtained from Figure 3. By combining wavelet transform denoising with sparse representation, the result of anomaly target detection is obviously better than the classical RX algorithm and
KRX algorithm. The results show that the WTSR algorithm detects 35 anomaly targets with 226 targets and 174 false alarms, the KRX algorithm detects 34 anomaly targets with 199 targets and 201 false alarms, the RX algorithm detects 23 anomaly targets with 159 targets and 241 false alarms. Through quantitative comparison, it can be found that the proposed WTSR algorithm is superior to other anomaly targets detection algorithms, it can also be found that the false alarm rate is reduced, and the detection accuracy of the algorithm is significantly improved.

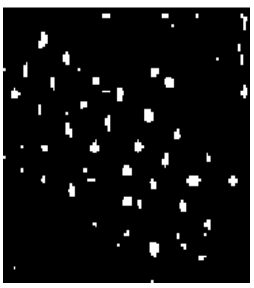

(a)

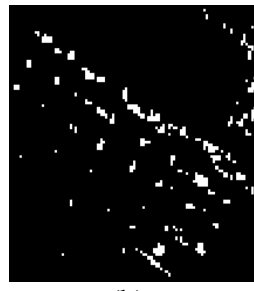

(b)

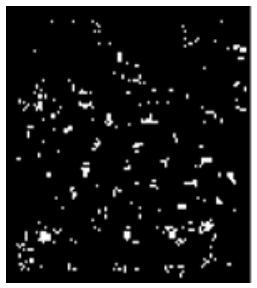

(c)
Figure 3. Anomaly detection results of three algorithms (a) WTSR algorithm, (b) KRX algotirhm, (c) RX algorithm

\section{Conclusion}

Anomaly detection algorithm of hyperspectral image has been studied for decades, which is necessary to continue theoretical analysis and introduce new signal processing methods because the current research is far from mature level. In paper, the spectral and spatial of hyperspectral image characteristics are analyzed, its noise is filtered by wavelet transform, and its anomaly target is detected by sparse representation theory. The results show that the algorithm is reasonable.

\section{Acknowledgment}

This study was supported by the National Natural Science Foundation of China under Grant No.61571145, and by Ministry of China Education Joint Fund for Production and Education No.2017B00001.

\section{References}

1. Reed I.S., Yu X., Adaptive multiple-band cfar detection of an optical pattern with unknown spectral distribution, IEEE Transactions on Acoustics, Speech and Signal Processing, 38, 10(1990).

2. Borghys D., Kåsen I., Achard V., et al, Comparative evaluation of hyperspectral anomaly detectors in different types of background. Algorithms \& Technologies for Multispectral Hyperspectral \& Ultraspectral Imagery, International Society for Optics and Photonics, San Diego, 12(2012).

3. A.P. Schaum, Hyperspectral anomaly detection beyond RX, Proc. SPIE, 2007.

4. Kwon H., Nasrabad N.M., Kernel RX-algorithm: A nonlinear anomaly detector for hyperspectral imagery, IEEE Transactions on Geoscience and 
Remote Sensing, 43,2(2005).

5. Kwon H., Nasrabadi N.M., A comparative study of kernel spectral matched signal detectors for hyperspectral target detection, USA:SPIE The International Society for Optical Engineering, 2005.

6. Yuan Zongze, Sun Hao, Ji Kefeng, et al, Local Sparsity Divergence for Hyperspectral Anomaly Detection, IEEE Geoscience and Remote Sensing Letters, 11, 10(2014).

7. Zhang Lili, Zhao Chunhui, Cheng Baozhi, A joint kernel collaborative representation based approach for hyperspectral image anomaly target detection, Journal of Optoelectronics-laser, 11(2015).

8. Lili Zhang, Baozhi Cheng, Yuwei Deng, A tensor-based adaptive subspace detector for hyperspectral anomaly detection, International Journal of Remote Sensing, 39, 8(2018).

9. Zhang Hanling, Application of MATLAB in image processing, Tsinghua University Press, 2008.

10. Song Xiangfa, Jiao Licheng, Hyperspectral remote sensing image based on sparse representation and spectral Information, Journal of Electronics \& Information Technology, 34, 2(2012).

11. Zhao Chunhui, Jing Xiaohao, Li Wei, Hyperspectral imagery target detection algorithm based on StOMP sparse representation, Journal of Harbin Engineering University, 36, 7(2015). 